\title{
Costs of conservative management of early-stage prostate cancer compared to radical prostatectomy-a claims data analysis
}

Alina Brandes ${ }^{1}$, Florian Koerber ${ }^{1}$, Larissa Schwarzkopf ${ }^{1}$, Matthias Hunger ${ }^{1}$, Wolf H. Rogowski ${ }^{1,2^{*}}$ and Raphaela Waidelich ${ }^{3}$

\begin{abstract}
Background: Due to widespread PSA testing incidence rates of localized prostate cancer increase but curative treatment is often not required. Overtreatment imposes a substantial economic burden on health care systems. We compared the direct medical costs of conservative management and radical therapy for the management of early-stage prostate cancer in routine care.

Methods: An observational study design is chosen based on claims data of a German statutory health insurance fund for the years 2008-2011. Three hundred fifty-three age-matched men diagnosed with prostate cancer and treated with conservative management and radical prostatectomy, are included. Individuals with diagnoses of metastases or treatment of advanced prostate cancer are excluded.

In an excess cost approach direct medical costs are considered from an insured community perspective for in- and outpatient care, pharmaceuticals, physiotherapy, and assistive technologies. Generalized linear models adjust for comorbidity by Charlson comorbidity score and recycled predictions method calculates per capita costs per treatment strategy.

Results: After follow-up of 2.5 years per capita costs of conservative management are $€ 6611$ lower than costs of prostatectomy ([-9734;-3547], $p<0.0001)$. Complications increase costs of assistive technologies by $30 \%(p=0.0182)$, but do not influence any other costs. Results are robust to cost outliers and incidence of prostate cancer diagnosis. The short time horizon does not allow assessing long-term consequences of conservative management.

Conclusions: At a time horizon of 2.5 years, conservative management is preferable to radical prostatectomy in terms of costs. Claims data analysis is limited in the selection of comparable treatment groups, as clinical information is scarce and bias due to non-randomization can only be partly mitigated by matching and confounder adjustment.
\end{abstract}

Keywords: Prostate cancer, Cost and cost analysis, Radical prostatectomy, Conservative management

\footnotetext{
* Correspondence: rogowski@helmholtz-muenchen.de

'Institute of Health Economics and Health Care Management, Helmholtz

Zentrum München, Neuherberg, Germany

${ }^{2}$ Institute of Public Health and Nursing Research, Health Sciences, University

of Bremen, Bremen, Germany

Full list of author information is available at the end of the article
} 


\section{Background}

Prostate cancer (PCa) is the second most common cancer in men worldwide and the most common in Germany $[1,2]$. Annual health care spending due to PCa is substantial and accounts for $€ 5.43$ billion in the European Union (EU). Germany exhibits the highest PCa-related health care costs per person in the EU, mostly due to inpatient expenditures [3]. Due to widespread prostate specific antigen (PSA) testing incidence rates and health care spending will increase significantly in the EU in the future. Detected tumors are often localized, clinically insignificant cancers, though. Data suggest that many men with localized PCa do not benefit from curative treatment with radical prostatectomy (RP) in terms of survival because tumor progression is so slow that no treatment is required [4].

The conservative management (CM) strategies active surveillance (AS) and watchful waiting (WW) are proposed in this context for the treatment of localized PCa to reduce overtreatment and subsequent complications such as erectile dysfunction (ED) and urinary incontinence (IC). Under AS regular biopsies, PSA tests, and digital rectal examinations are performed to initiate curative treatment if tumor progression occurs. If life expectancy is less than 10 years or comorbidity does not allow any other form of PCa-treatment WW is recommended. This strategy has no standardized follow-up scheme; symptomoriented, palliative therapy is initiated if disease progresses [5]. A CM-strategy may save health care costs of unnecessary curative therapy and treatment of its adverse effects.

Current economic studies suggest that, while health outcome in terms of survival and quality of life is similar for $\mathrm{CM}$ and curative treatment, $\mathrm{CM}$ is a cost-saving strategy over the first 5 to 10 years of treatment [6-12]. Most studies base cost analysis on US reimbursement values, which are often not representative for European health care [13]. To guide treatment decisions in a European health care context observational studies are needed displaying routine care and actual health care spending on CM compared to RP.

In a German health care context, claims data of statutory health insurance (SHI) funds are a valuable evidence source as medical care, resource use, and costs are documented in detail and over a long period of time for a large cohort of patients. About $85 \%$ of the German population is insured within the social security system of SHI, which is characterized by pay-as-you-go financing and incomedependent insurance contributions. The remaining 15\% are covered by private insurance. SHI covers inpatient and outpatient care, pharmaceuticals, physiotherapy, and assistive technologies. Co-payments of patients are compulsory, especially for pharmaceuticals and medical aids [14].

It is the objective of this paper to analyze claims data of a German AOK SHI fund regarding costs of CM compared to RP in an age-matched and comorbidityadjusted cohort of men with early-stage PCa.

\section{Methods \\ Data}

AOK Baden-Württemberg is the largest SHI fund in the south-western German federal state of BadenWürttemberg with about 3.8 million insured individuals. About $25 \%$ of the insured population is retired and over 65 years. Data on all claims incurred at AOK between 2008 and 2011 were provided on a patient level. Co-payments to medical services covered by SHI are included in the dataset, whereas patients' out-ofpocket payments for other services are not. German data protection laws were considered during extraction and analysis of data and AOK approved of the intended use of the data. An ethics committee was consulted regarding this study; ethics approval is not necessary as identification of individuals is not possible in the dataset.

\section{Study design and cohort selection}

A prospective, longitudinal study design is chosen, where a cohort of men diagnosed with early-stage PCa is followed from the point of treatment initiation.

The study period is January 1st 2008 to December 31st 2011 and is divided into three sections (Fig. 1):

1) The 6-month pre-observation period (January 1st 2008 to June 30th 2008) is created as a basis for the calculation of the Charlson comorbidity score (CCS), in order to allow for comorbidity adjustment of costs.

2) In the 12-month observation period from July 1 st 2008 to June 30th 2009 PCa-cases are identified and categorized into the two treatment-groups $\mathrm{CM}$ and RP.

3) Both treatment groups are followed-up for a period of exactly 2.5 years (follow-up period). In case of RP, follow-up time starts individually for each person after the date of the initial prostatectomy procedure and follows each man for 2.5 years on an individual basis. Men under CM, on the other hand, are followed for a fixed period from July 1st 2009 to December 31st 2011. The reason for this is, firstly, that the starting point of CM cannot be established in the cohort; an artificial starting point has to be created, which is the beginning of the observation period (July 1st 2008). Secondly, follow-up is not intended to start with the onset of CM. This is due to the cohort selection, where men under CM have to be surveyed for at least 12 months (the observation period) to be included in the cohort; men dying in this period are not considered for analysis. In the RP- group a single event in the observation period determines inclusion in the cohort, which in turn possibly 


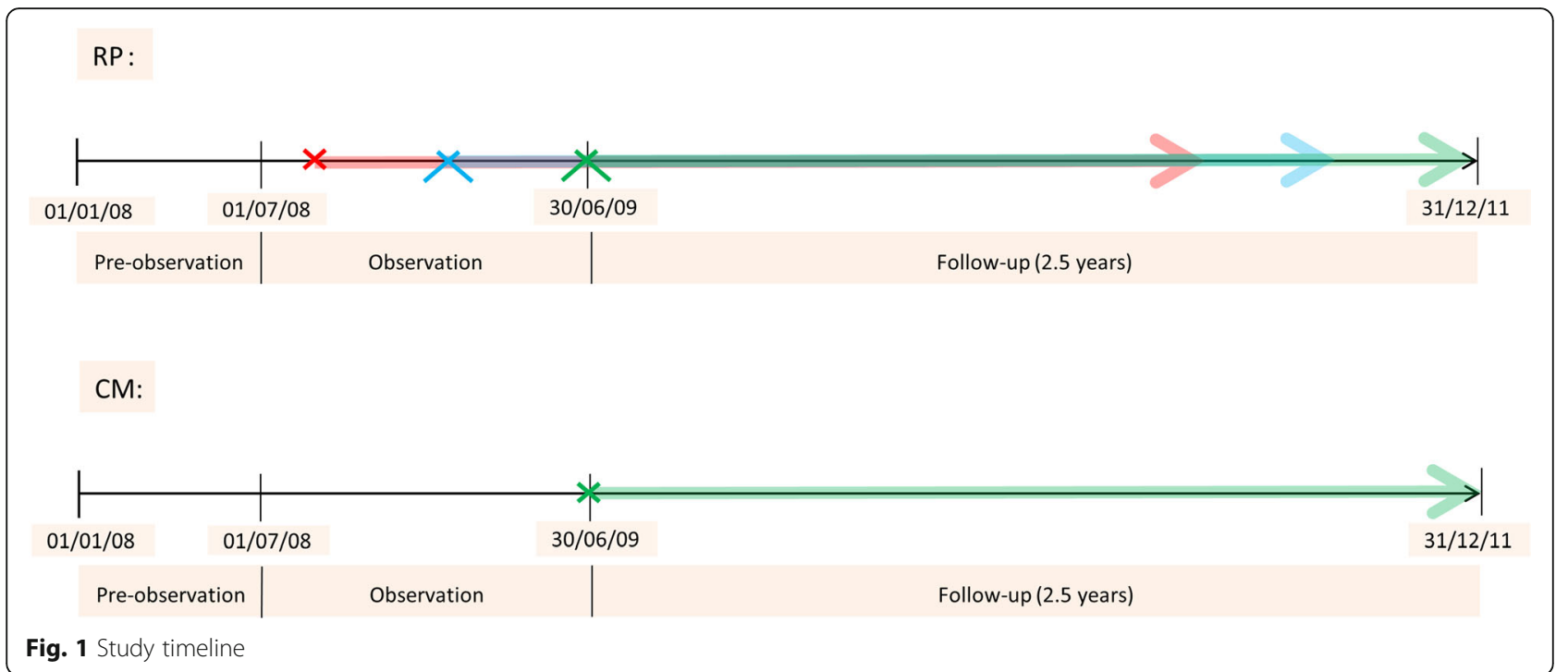

includes men dying in this period. To account for this bias, follow-up of AS is offset by 12 months.

The baseline dataset includes all men with an ICD-10 diagnosis 'C61-Malignant neoplasm of prostate' in the 12-month observation period. PCa diagnosis is validated by only considering men with at least one inpatient or at least two outpatient diagnostic codes $(N=25,367)$. After exclusion of 71 individuals not constantly insured at AOK Baden-Württemberg in the study period (defined by the coding of 'transition to another insurance fund' in the claims data) and three individuals with female gender coding, the baseline data set includes 25,293 individuals (Fig. 2, Additional file 1: Table S1 and Table S2).

$\mathrm{CM}$ is defined by outpatient procedure codes of at least two PSA tests and one prostate biopsy during the observation period, including AS- and WW-patients [15]. Additionally, patients undergoing any form of PCa-specific therapy other than CM are excluded to avoid misclassification of patients due for curative treatment as $\mathrm{CM}$ cases. In the follow-up period, men in the CM-group do not have to be surveyed as defined in the observation period; they may move on to any other form of PCa-specific therapy.

$\mathrm{RP}$ is defined by procedure codes on open, laparoscopic, or robotic-assisted radical prostatectomy. Patients with metastatic disease or any other cancer disease coding are excluded. Exclusion of any PCa-specific therapy other than $\mathrm{RP}$ is not necessary, as these are not found in the RPgroup.

Considering the inclusion criteria the cohort consists of 124 and 910 individuals under CM and RP, respectively.

\section{Statistical analysis}

To control for age as a confounder of cost differences, treatment groups are matched by $+/-2$ years. After matching in a ratio of 1:2 the CM-group includes 107 individuals, and the RP-group includes 214 individuals.

Patients' age and CCS as well as prevalence of ED, IC, and benign prostate hyperplasia (BPH) are calculated at baseline before and after matching. For calculation of CCS the comorbidity-group 'cancer' is set 0, because diseases other than PCa are of interest for adjustment.

We use an excess cost approach to display cost differences between treatment strategies for inpatient and outpatient care, pharmaceuticals, physiotherapy, and assistive technologies. All direct medical costs and co-payments are considered that are relevant for the perspective of the SHI scheme insured community ( $\$ 35 b$ (1) SGBV) [16]. Comorbidity-adjusted cost differences between treatment groups are estimated by a generalized linear model (GLM) with a gamma distribution and log link to account for the typically skewed distribution of cost data. If less than $10 \%$ of individuals in the cohort have zero costs a small amount of 1 Euro $(€)$ is assigned to include them in the analysis [17]. If individuals with zero costs account for more than $10 \%$ of the cohort a two-part model is used: at first, the probability of health care expenditure is predicted with a logistic regression model. Secondly, costs are estimated by a GLM, as described above, conditional for nonzero costs. To derive unconditional costs the probability of expenditure is multiplied by the predicted conditional costs [18]. Recycled predictions are used to estimate per capita costs per treatment strategy in addition to percentage values of differences reported by the GLM [19]. All costs are rounded to the nearest $€$ to present only full $€$ amounts with no cent values; discounting is not considered due to the short study period.

Ninety-five percent confidence intervals (CI) are calculated for costs via a non-parametric bootstrap approach based on 1000 replications. Difference in costs is tested 


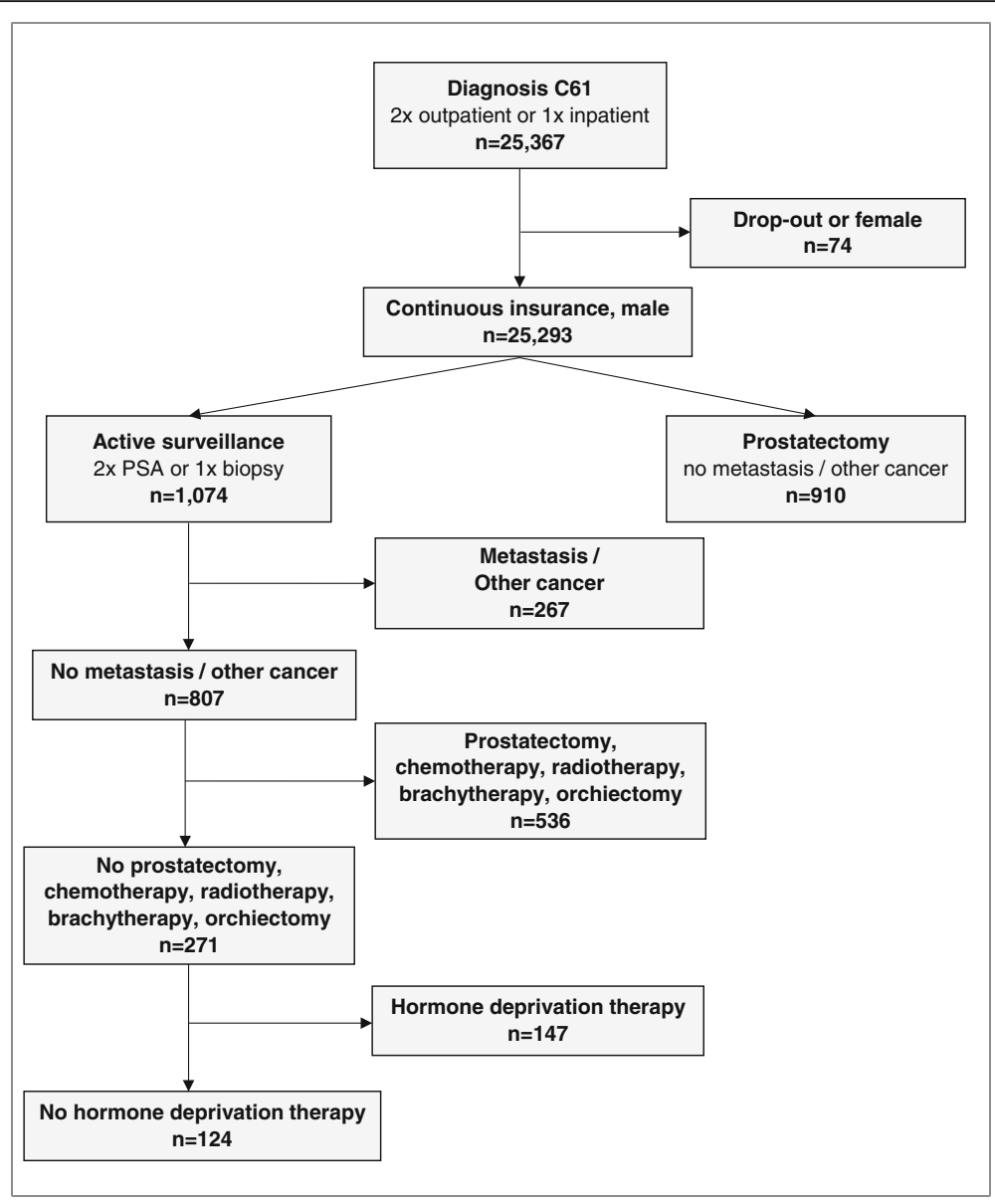

Fig. 2 Cohort selection

via bootstrap hypothesis testing and $p$-values less or equal than 0.05 are considered statistically significant [20]. The CCS is included in the regression models as a continuous variable $[21,22]$. To additionally estimate the influence of ED and IC on treatment costs, complication is included in the regression models as a binary variable. Extended models with an interaction between treatment strategy and CCS do not improve model fit.

In a sensitivity analysis we include only incident $\mathrm{PCa}$ cases, with no C61 code in the pre-observation period-assuming that $\mathrm{CM}$ is initiated with the first $\mathrm{PCa}$-diagnosis-to estimate the influence of the cohort selection on outcomes.

Statistical analyses are performed with the software package SAS, version 9.3.

\section{Results}

\section{Descriptive analysis}

Mean age at baseline after matching is 69 years (STD 6.80) in the RP-group and 70 years (STD 7.13) in the CMgroup, with a mean CCS of 0.11 and 0.19 , respectively. $\mathrm{ED}, \mathrm{IC}$ and $\mathrm{BPH}$ are most prevalent in the RP-group at baseline (Table 1).

\section{Cost analysis}

Figure 3 shows unadjusted per capita costs for the two treatment strategies grouped by inpatient and outpatient treatment, pharmaceuticals, physiotherapy, and assistive technologies. RP has overall higher mean costs, when compared to CM. Comorbidity-adjusted analysis confirms this (RP: €18,544, CM: €11,933).

Comparison of adjusted costs (Table 2) displays that CM has significantly lower mean inpatient (€-5845

Table 1 Baseline characteristics before and after matching

\begin{tabular}{llllll}
\hline & \multicolumn{2}{l}{ Before matching } & & \multicolumn{2}{l}{ After matching } \\
\cline { 2 - 3 } \cline { 6 - 7 } & RP & CM & & RP & CM \\
\cline { 1 - 3 } Total (n) & 910 & 124 & & 214 & 107 \\
Age (mean, STD) & $66(6.64)$ & $70(8.31)$ & & $69(6.80)$ & $70(7.13)$ \\
CCS (mean, STD) & $0.13(0.71)$ & $0.19(0.62)$ & & $0.11(0.63)$ & $0.19(0.63)$ \\
ED (n, prop.) & $79(0.09)$ & $5(0.04)$ & & $24(0.11)$ & $5(0.05)$ \\
IC (n, prop.) & $18(0.02)$ & $5(0.04)$ & & $10(0.05)$ & $3(0.03)$ \\
BPH (n, prop.) & $511(0.56)$ & $83(0.67)$ & & $164(0.77)$ & $73(0.68)$ \\
\hline
\end{tabular}

$C M$ conservative management, $n$ number, prop. proportion, $R P$ radical prostatectomy, STD standard deviation 


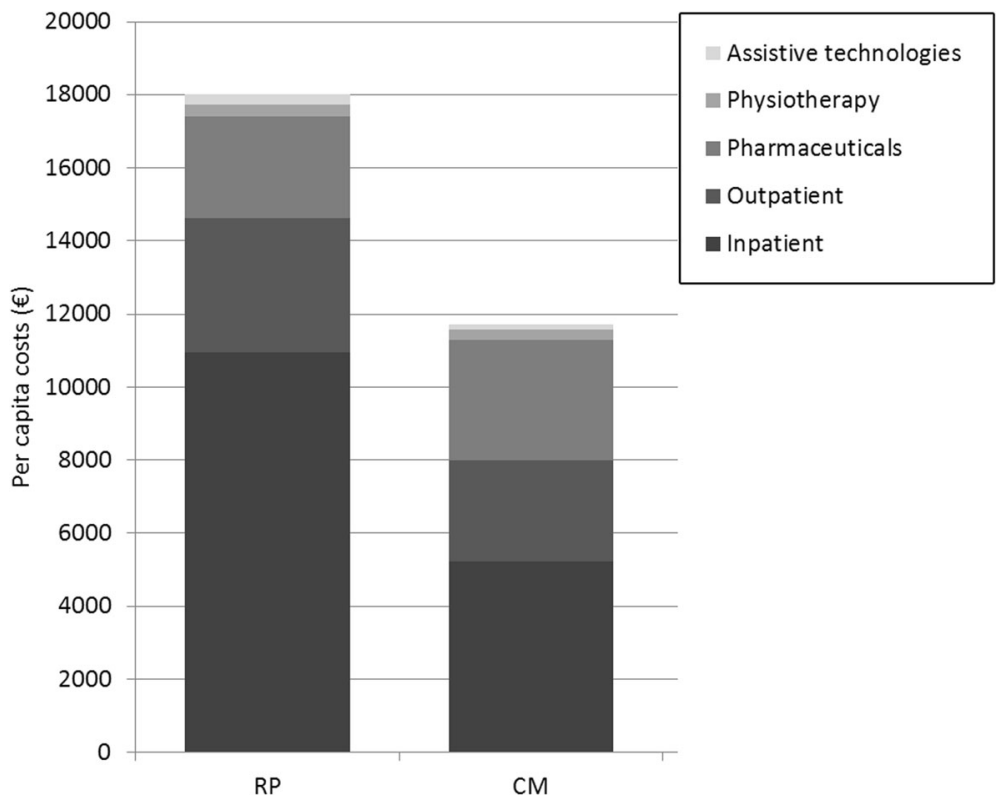

Fig. 3 Costs per capita (€)-unadjusted

[-7632;-3895], $p<0.0001)$ and outpatient costs $(€-961$ [-1622;-361], $p=0.002)$ as well as costs for assistive technologies $(€-141 \quad[-230 ;-50], p=0.006)$ than RP. Overall, total incremental costs of $\mathrm{CM}$ are significantly lower compared to RP ( $€-6611$ [-9734;-3547], $p<0.0001$ ) (Fig. 4). Inclusion of complication as a binary variable in the regression models does not change cost differences between treatment strategies. Concerning total costs there is no significant cost difference found between individuals with and without complications. In case of assistive technologies a significant $30 \%$ increase in costs for individuals with complications is estimated $(p=0.018)$.

For the analysis of inpatient, outpatient, pharmaceutical, physiotherapy, and assistive technology costs two-part models are used, as more than $10 \%$ of individuals have zero costs respectively. Total costs are analyzed in a one-part model, as no individuals have zero costs.

Table 2 Difference in costs $(€)$ between CM and RP-adjusted

\begin{tabular}{llll}
\hline & \multicolumn{2}{l}{ CM-RP } & \\
\cline { 2 - 4 } & Mean & $95 \% \mathrm{Cl}$ & $P$-value \\
\hline Inpatient & -5845 & -7632 to -3895 & $<0.0001$ \\
Outpatient & -961 & -1622 to -361 & 0.002 \\
Pharmaceuticals & 587 & -556 to 1718 & 0.274 \\
Physiotherapy & -58 & -214 to 114 & 0.460 \\
Assistive technologies & -141 & -230 to -50 & 0.006 \\
Total costs & -6611 & -9734 to -3547 & $<0.0001$ \\
\hline
\end{tabular}

$C M$ conservative management, $R P$ radical prostatectomy

\section{Sensitivity analysis}

Analysis of costs is repeated including incident PCa-cases only, which comprised of an age-matched cohort of 320 men (CM: 64, RP: 128). Cost analysis reveals that mean comorbidity-adjusted total costs of CM increase by $€ 1496$ to $€ 13,430$ in total compared to base case. Outpatient costs of $\mathrm{CM}$ also increase compared to base case from $€ 2332$ to $€ 2499$. Overall, CM is still significantly less costly than RP $(€-5263$ [-9530;-1164], $p=0.016)$ in the incident cohort.

\section{Discussion}

This is, to our knowledge, the first observational study comparing costs of CM to RP in a European health care context. Use of claims data allows picturing actual treatment practice of early-stage $\mathrm{PCa}$ in Germany. Cost analysis is based on exact and detailed cost information on different health care sectors which is representative for the costs incurred by the SHI scheme insured community, contrary to many clinical trials. Compared to modeling studies assumptions on resource use and reimbursement practice are not required.

$\mathrm{CM}$ is overall significantly less costly than RP which mainly originates in the significant difference of inpatient costs, presumably due to the high costs of the initial RPsurgery. Compared to RP outpatient care is also less costly under $\mathrm{CM}$ in the period of 2.5 years follow-up, despite that the main costs of CM arise in outpatient care. This is due to different cost patterns, where surgery incurs high initial outpatient costs (e.g. for post-operative care after $\mathrm{RP}$ ) while costs of surveillance, especially AS, are more equally distributed over time [6-9]. The sensitivity 


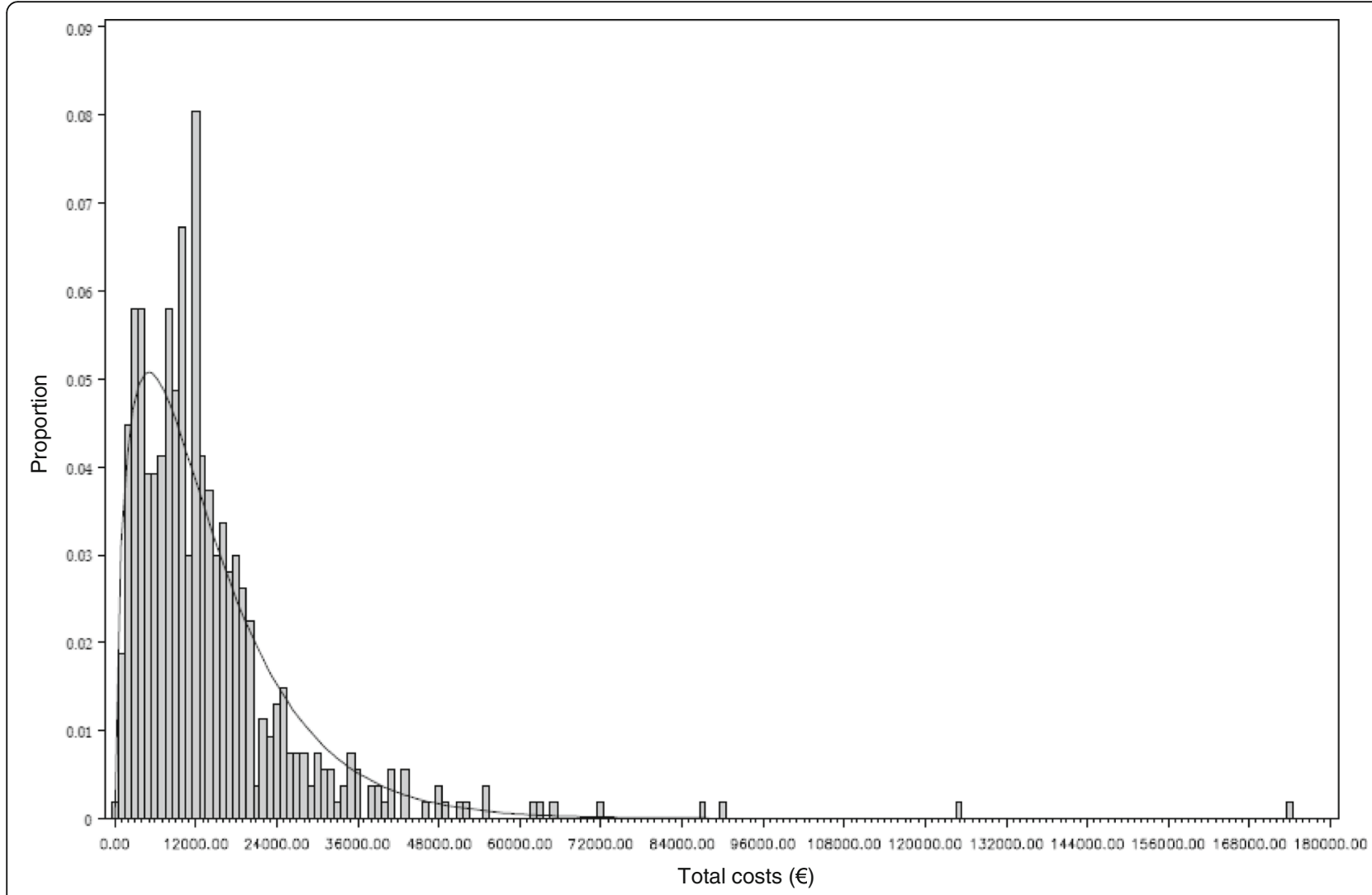

Fig. 4 Histogram total costs

analysis with incident $\mathrm{PCa}$-cases-which assumes that $\mathrm{CM}$ is initiated with the first PCa-diagnosis-shows that outpatient costs increase compared to base case which may lead to the conclusion that $\mathrm{CM}$-costs are comparably higher in the initial phase.

In the base case analysis pharmaceutical costs are higher under $\mathrm{CM}$ compared to RP, not significantly though; this may be explained by pharmaceutical treatment of $\mathrm{BPH}$ in the CM-group which is not necessary in the RP-group.

Published cost studies with a European health care context comparing CM and RP report similar cost differences to our study. Andersson et al. [23] report €6123 lower costs of WW compared to RP over a follow-up of 12 years based on data from the SPCG-4 clinical trial [23]. In a lifetime modeling study, Koerber et al. [7] estimate that under AS compared to RP costs are reduced by about $€ 6900$ [7]. Both in the published studies and in our study, the cost difference between CM and RP is almost exclusively due to the costs of the initial RP-surgery.

Several US-based observational and modeling studies show that CM is the least costly strategy over the whole study duration, consistent with the results presented here $[6,10,12,8,24]$. Only the study by Perlroth et al. [11] reports that from year 2 of the study on costs of
CM become equal to costs of RP [11]. Perlroth et al. do not state unit costs of the surveillance scheme; however, other US-based studies show that the unit costs of prostate biopsy (\$605-\$1102) alone are considerably higher in the US health-care context than unit costs of the whole surveillance scheme (PSA testing and biopsy) in Germany $(€ 44)[6,8,24]$. Because of the limited study duration only short-term costs can be assessed in this study; as published studies do, however, suggest that cost differences between $\mathrm{CM}$ and curative treatment, especially RP, arise in the first years after treatment, the results of this study may support the conclusion that costs of CM do not arise to costs of RP in a lifetime perspective [25].

Our study has some limitations. Regarding cohort selection, there is no information on tumor stage or Gleason score included in claims data, which might allow clinical classification of PCa. To account for this limitation we define early-stage PCa as absence of both diagnoses of metastases and treatment associated with recurrence or advanced tumor progression. Inclusion in the CM-group cannot be based on clinical criteria or specific procedure codes and therefore a distinction between WW- and AS-patients is not possible. Seven Percent of men under CM receive at least one biopsy during follow-up which suggests that these are under 
AS, while the remaining men may be under WW. The cohort's life expectancy, however, is with a mean age of 70 years at baseline greater than 10 years and men areaccording to treatment guideline-not eligible to WW. Patients might actually be under AS, but are not surveyed by regular biopsies according to guideline. This might be due to current studies on adverse effects of serial biopsies on erectile function and infectious complications [26, 27]. Furthermore, randomization of individuals into treatment groups is not possible as in any other observational study; estimated differences between groups might be attributed to unequal distribution of confounding variables. This bias is reduced by matching and regression analysis only to a certain degree, because the number of variables available for confounder adjustment is limited in our data set. Also, descriptive analysis of patient characteristics after matching shows that men in the CM-group have more co-morbidities than in the RPgroup (CCS 0.19 vs. 0.11), which is adjusted for in regression analysis. Men in the RP-group, on the other hand, show higher rates of $\mathrm{BPH}$ than men under $\mathrm{CM}$, which might be explained by the treatment itself; radical prostatectomy is a treatment for $\mathrm{BPH}$, too.

Regarding the study design, a 12-month observation period is chosen which excludes CM-patients who receive curative therapy in this period. The probability for curative treatment in the first year of $\mathrm{CM}$ is, however, low $(<5 \%)$ [28]. Also it is necessary in this study to allow enough time to confirm the CM-strategy and exclude patients who are waiting for curative treatment, which is done 6 months after the initial PCa-diagnosis latest [29]. This study might underestimate costs of the CM-strategy slightly by the exclusion of cases with curative treatment in the first year. Alternative treatment options like radiotherapy were excluded from the study due to the availability of claims data for a limited duration. Complications after radiotherapy, usually develop after a longer period than analyzed here, which might underestimate the costs of radiotherapy.

Regarding cost analysis, calculation of costs for single procedures is not conducted in this study as case numbers are too small to be representative. CCS is not able to adjust for comorbidities with high outpatient and pharmaceutical costs as the score was developed to assess inpatient mortality. We do not account for dependency of data due to matching by using conditional regression analysis. Dependency of data is very low as we only match for age; also no longitudinal analysis of single individuals is intended.

Generalizability of results to the general population is limited as claims data cannot report out-of-pocketpayments. Also, due to the excess cost approach absolute costs do not reflect $\mathrm{PCa}$ treatment specific costs and no conclusion can be inferred on the costs of RP and CM from this study.

\section{Conclusions}

Overall, our analysis indicates that initial treatment costs as well as short-term follow-up costs of CM based on claims data of a German SHI fund are significantly lower than the costs of curative therapy with RP for early-stage $\mathrm{PCa}$-predominantly due to the high initial costs of the surgery. Treatment of complications following initial therapy has a very small impact on costs. These results must be interpreted in light of the limitations regarding cohort selection in claims data.

Further research is necessary to analyze costs of CM compared to curative treatment over a longer time horizon than reported in this study including costs of long-term complications and end-of-life care.

\section{Additional file}

Additional file 1: Table S1. Diagnostic codes for cohort selection. Table S2. Procedure codes for cohort selection. (DOC 34 kb)

\section{Abbreviations}

AS: Active surveillance; BPH: Benign prostate hyperplasia; CCS: Charlson comorbidity score; Cl: Confidence intervals; CM: Conservative management; ED: Erectile dysfunction; EU: European Union; GLM: Generalized linear model; IC: Urinary incontinence; PCa: Prostate cancer; PSA: Prostate specific antigen; RP: Radical prostatectomy; SHI: Statutory health insurance; WW: Watchful waiting

\section{Acknowledgements}

We wish to thank all involved project partners for helping to retrieve the original data set and for clarifying questions regarding the data. The authors are grateful for the assistance of Frauke Hörnig in preparing the dataset for analysis.

\section{Funding}

The research is carried out on behalf of the Helmholtz Zentrum München. The Helmholtz Zentrum München is an independent organization funded by the German and Bavarian government. Research for this paper was supported by a grant from the German Federal Ministry of Education and Research (grant number 01KQ0902V). The authors do not have a conflict of interest with regard to this project. Publication of study results was not contingent on the funding party's approval or censorship of the manuscript.

\section{Availability of data and materials}

The authors confirm that the data utilized in this study cannot be made available in the manuscript, the supplemental files, or in a public repository due to German data protection laws ('Bundesdatenschutzgesetz', BDSG). Therefore, they are stored on a secure drive in the senior author's institution to facilitate replication of the results. Generally, access to data of statutory health insurance funds for research purposes is possible only under the conditions defined in German Social Law (SGB V § 287). Requests for data access can be sent as a formal proposal specifying the recipient and purpose of the data transfer to the appropriate data protection agency. Access to the data used in this study can only be provided to external parties under the conditions of the cooperation contract of this research project and after written approval by the sickness fund. For assistance in obtaining access to the data, please contact the corresponding author, Prof. Dr. Wolf $\mathrm{H}$. Rogowski, at rogowski@uni-bremen.de.

\section{Authors' contributions}

$\mathrm{AB}$ conducted the analysis and wrote the manuscript. FK provided input on the costs of RP. LS provided input on issues related with analysis of claims data. MH provided statistical advice. WR conceived the study and provided inputs on various aspects including study design, data acquisition and writing the manuscript. RW supervised the study from a medical perspective. All authors were involved in writing the manuscript and read and approved the final version. 


\section{Competing interests}

The authors declare that they have no competing interests.

\section{Consent for publication}

Consent to publish is not applicable since the manuscript does not contain individual person's data.

\section{Ethics approval and consent to participate}

The ethics committee of the Bavarian state's medical association was consulted on November $21^{\text {st }} 2013$ regarding the need of a formal ethics approval for this study. The ethics committee replied on November 22nd 2013 that formal approval is not necessary as identification of individuals is not possible in the dataset (Reference number: 2013-108).

According to $\$ 75$ Social Code Book 10 it is not necessary to obtain consent to participate in claims data based studies where it is not reasonable to achieve this. This is the case in this study due to the large number of insurants needed to conduct the study. The state ministry of Baden-Württemberg supervising the compliance with $\$ 75$ Social Code Book 10 approved this study on September 17th 2012 (Reference number: 52-5227-1-027)

\section{Author details}

'Institute of Health Economics and Health Care Management, Helmholtz Zentrum München, Neuherberg, Germany. ${ }^{2}$ Institute of Public Health and Nursing Research, Health Sciences, University of Bremen, Bremen, Germany. ${ }^{3}$ Department of Urology, Ludwig-Maximilian University, Munich, Germany.

Received: 19 January 2016 Accepted: 29 October 2016

\section{Published online: 18 November 2016}

\section{References}

1. Ferlay J, Soerjomataram I, Ervik M, Dikshit R, Eser S, Mathers C, et al. GLOBOCAN 2012, Cancer Incidence and Mortality Worldwide: IARC CancerBase No. 11. Lyon: International Agency for Research on Cancer. 2013. http://globocan.iarc.fr. Accessed 13 Aug 2014

2. Kraywinkel K, Bertz J, Laudi A, Wolf U. Epidemiologie und Früherkennung häufiger Krebserkrankungen in Deutschland. GBEkompakt. Berlin: Robert Koch-Institut: 2012.

3. Luengo-Fernandez R, Leal J, Gray A, Sullivan R. Economic burden of cancer across the European Union: a population-based cost analysis. Lancet Oncol. 2013;14(12):1165-74. doi:10.1016/s1470-2045(13)70442-x.

4. Popiolek M, Rider JR, Andrén O, Andersson S-O, Holmberg L, Adami H-O, et al. Natural history of early, localized prostate cancer: a final report from three decades of follow-up. Eur Urol. 2013;63(3):428-35. http://dx.doi.org/10. 1016/j.eururo.2012.10.002

5. Mottet N, Bastian PJ, Bellmunt J, Van den Bergh RC, Bolla M, Van Casteren NJ, et al. Guidelines on prostate cancer. 2014.

6. Keegan KA, Dall'Era MA, Durbin-Johnson B, Evans CP. Active surveillance for prostate cancer compared with immediate treatment: an economic analysis. Cancer. 2012;118(14):3512-8. doi:10.1002/cncr.26688.

7. Koerber F, Waidelich R, Stollenwerk B, Rogowski W. The cost-utility of open prostatectomy compared with active surveillance in early localised prostate cancer. BMC Health Serv Res. 2014;14:163. doi:10.1186/1472-6963-14-163.

8. Hayes JH, Ollendorf DA, Pearson SD, Barry MJ, Kantoff PW, Lee PA. Observation versus initial treatment for men with localized, low-risk prostate cancer: a costeffectiveness analysis. Ann Intern Med. 2013;158:853-60.

9. Corcoran AT, Peele PB, Benoit RM. Cost comparison between watchful waiting with active surveillance and active treatment of clinically localized prostate cancer. Urology. 2010;76(3):703-7. doi:10.1016/j.urology.2009.12.071.

10. Snyder CF, Frick KD, Blackford AL, Herbert RJ, Neville BA, Carducci MA, et al. How does initial treatment choice affect short-term and long-term costs for clinically localized prostate cancer? Cancer. 2010;116(23):5391-9. doi:10. 1002/cncr.25517.

11. Perlroth DJ, Bhattacharya J, Goldman DP, Garber AM. An economic analysis of conservative management versus active treatment for men with localized prostate cancer. J Natl Cancer Inst Monogr. 2012;2012(45):250-7. doi:10. 1093/jncimonographs/lgs037.

12. Wilson LS, Tesoro R, Elkin EP, Sadetsky N, Broering JM, Latini DM, et al. Cumulative cost pattern comparison of prostate cancer treatments. Cancer. 2007;109(3):518-27. doi:10.1002/cncr.22433.

13. Roehrborn CG, Black LK. The economic burden of prostate cancer. BJU Int 2011;108(6):806-13. doi:10.1111/j.1464-410X.2011.10365.X.
14. Busse R, Riesberg A. Health care systems in transition: Germany Copenhagen: WHO Regional Office for Europe on behalf of the European Observatory on Health Systems and Policies; 2004.

15. Leitlinienprogramm Onkologie (Deutsche Krebsgesellschaft, Deutsche Krebshilfe, AWMF). Interdisziplinäre Leitlinie der Qualität S3 zur Früherkennung, Diagnose und Therapie der verschiedenen Stadien des Prostatakarzinoms Langversion 3.1 AWMF Registernummer: 034/022OL. 2014. http://leitlinienprogramm-onkologie.de/uploads/tx_sbdownloader/LL_ Prostata_Langversion_3.1.pdf. Accessed 06 Jan 2015.

16. Institute for Quality and Efficiency in Health Care (IQWiG). Working Paper Cost Estimation. 2009.

17. Barber J, Thompson S. Multiple regression of cost data: use of generalised linear models. J Health Serv Res Policy. 2004;9(4):197-204. doi:10.1258/ 1355819042250249

18. Buntin MB, Zaslavsky AM. Too much ado about two-part models and transformation?: Comparing methods of modeling Medicare expenditures. J Health Econ. 2004;23(3):525-42. http://dx.doi.org/10.1016/j.jhealeco.2003.10.005.

19. Graubard BI, Korn EL. Predictive margins with survey data. Biometrics. 1999; 55(2):652-9. doi:10.2307/2533821.

20. Afifi AA, Kotlerman JB, Ettner SL, Cowan M. Methods for improving regression analysis for skewed continuous or counted responses. Annu Rev Public Health. 2007:28:95-111. doi:10.1146/annurev.publhealth.28.082206.094100.

21. Salonia A, Castagna G, Saccà A, Ferrari M, Capitanio U, Castiglione F, et al. Is Erectile Dysfunction a Reliable Proxy of General Male Health Status? The Case for the International Index of Erectile Function-Erectile Function Domain. J Sex Med. 2012;9(10):2708-15. doi:10.1111/j.1743-6109.2012.02869.x.

22. Hall WH, Ramachandran R, Narayan S, Jani AB, Vijayakumar S. An electronic application for rapidly calculating Charlson comorbidity score. BMC Cancer. 2004:4:94. doi:10.1186/1471-2407-4-94

23. Andersson SO, Andren O, Lyth J, Stark JR, Henriksson M, Adami HO, et al. Managing localized prostate cancer by radical prostatectomy or watchful waiting: Cost analysis of a randomized trial (SPCG-4). Scand J Urol Nephrol. 2011:45(3):177-83. doi:10.3109/00365599.2010.545075.

24. Eldefrawy A, Katkoori D, Abramowitz M, Soloway MS, Manoharan M. Active surveillance vs. treatment for low-risk prostate cancer: a cost comparison. Urol Oncol. 2013;31(5):576-80. doi:10.1016/j.urolonc.2011.04.005.

25. Manoharan M, Eldefrawy A, Katkoori D, Antebi E, Soloway MS. Comparison of urologist reimbursement for managing patients with low-risk prostate cancer by active surveillance versus total prostatectomy. Prostate Cancer Prostatic Dis. 2010;13(4):307-10. doi:10.1038/pcan.2010.34.

26. Braun K, Ahallal Y, Sjoberg DD, Ghoneim T, Dominguez Esteban M, Mulhall $J$, et al. Effect of repeated prostate biopsies on erectile function in men on active surveillance for prostate cancer. J Urol. 2014;191(3):744-9. http://dx. doi.org/10.1016/j.juro.2013.08.054.

27. Ehdaie B, Vertosick E, Spaliviero M, Giallo-Uvino A, Taur Y, O'Sullivan M, et al The impact of repeat biopsies on infectious complications in men with prostate cancer on active surveillance. J Urol. 2014;191(3):660-4. doi:10. 1016/j.juro.2013.08.088

28. Klotz L, Zhang L, Lam A, Nam R, Mamedov A, Loblaw A. Clinical results of long term follow-up of a large, active surveillance cohort with localized prostate cancer. J Clin Oncol. 2010;28(1):126-31. doi:10.1200/jco.2009.24.2180.

29. Potosky AL, Davis WW, Hoffman RM, Stanford JL, Stephenson RA, Penson $D F$, et al. Five-year outcomes after prostatectomy or radiotherapy for prostate cancer: the prostate cancer outcomes study. J Natl Cancer Inst. 2004;96(18):1358-67. doi:10.1093/jnci/djh259.

\section{Submit your next manuscript to BioMed Central and we will help you at every step:}

- We accept pre-submission inquiries

- Our selector tool helps you to find the most relevant journal

- We provide round the clock customer support

- Convenient online submission

- Thorough peer review

- Inclusion in PubMed and all major indexing services

- Maximum visibility for your research

Submit your manuscript at www.biomedcentral.com/submit 